K. Shiraiwa

Nagoya Math. J.

Vol. 66 (1977), 151-166

\title{
BOUNDEDNESS AND CONVERGENCE OF SOLUTIONS OF DUFFING'S EQUATION
}

\section{Dedicated to Professor Ryoji Shizuma on his 60-th birthday}

\section{KENICHI SHIRAIWA}

In this paper, we shall discuss boundedness of solutions of the equation

$$
x^{\prime \prime}+f(x) x^{\prime}+g(x)=e(t) \quad\left({ }^{\prime}=d / d t\right)
$$

under suitable conditions. And we shall discuss asymptotic stability of a periodic solution and convergence of solutions for the equation

$$
x^{\prime \prime}+c x^{\prime}+g(x)=e(t)
$$

for a positive constant $c$ and a periodic function $e(t)$ under some restricted conditions.

This work is motivated by the work of $\mathrm{H}$. Kawakami [3], which gives some numerical computations on the equation $x^{\prime \prime}+k x^{\prime}+x^{3}=B \cos t$ for positive constants $k$ and $B$. Kawakami's work contains many interesting informations on Duffing's equation of the above type, and this paper deals with only small part of his work. There is also very interesting experimental results by C. Hayashi, Y. Ueda and H. Kawakami [2]. Also, our paper heavily depends on the work of W. S. Loud [4].

\section{§1. The boundedness theorem}

The equation (2) is a special case of (1), and the equation (1) is equivalent to the following system of equations.

$$
\left\{\begin{array}{l}
x^{\prime}=y \\
y^{\prime}=-f(x) y-g(x)+e(t)
\end{array}\right.
$$

THEOREM 1. In the equation (1) we assume the following conditions

Received June 1, 1976. 
(a), (b) and (c).

(a) There exists a solution of (1) under any initial condition.

(b) There exist positive constants $c$ and $E$ such that

$$
f(x) \geqq c \quad \text { and } \quad|e(t)| \leqq E .
$$

(c) $g(x)$ is a differentiable function satisfying the following conditions (i), (ii) and (iii).

(i) $g^{\prime}(x)$ is bounded on any finite interval.

(ii) $g^{\prime}(x) \geqq 0$.

(iii) $\lim _{x \rightarrow \infty} g(x)>E$ and $\lim _{x \rightarrow-\infty} g(x)<-E$.

By the condition (c), $g(x)$ is a monotone increasing function, and there exist numbers $x_{1}$ and $x_{2}\left(x_{1}<x_{2}\right)$ such that

$$
g\left(x_{1}\right)=-E \text { and } g\left(x_{2}\right)=E .
$$

Let $x(t)$ be any solution of (1). Then there exists a number $t_{0}$ such that

$$
x_{1}-4 E / c^{2} \leqq x(t) \leqq x_{2}+4 E / c^{2}
$$

and

$$
\left|x^{\prime}(t)\right| \leqq 4 E / c \quad \text { for any } t \geqq t_{0} .
$$

Our proof is similar to that of Theorem 1 of W. S. Loud [4]. He assumed that $g^{\prime}(x) \geqq b$ for some positive constant $b$ in his paper and got an additional information. But the essential part of his argument is applicable in our case, too.

Proof of Theorem 1. From the equation (5) we obtain the following equation.

$$
y \frac{d y}{d x}=-f(x) y-g(x)+e(t)
$$

We consider the following equations.

$$
\begin{array}{ll}
y \frac{d y}{d x}=-c y-g(x)+E & (y \geqq 0) \\
y \frac{d y}{d x}=-c y-g(x)-E & (y \leqq 0)
\end{array}
$$


By our assumption (c) (i) the existence and uniqueness of solutions of (5) and (6) are guaranteed.

Also, the following inequalities hold by our assumption (b).

$$
\begin{array}{ll}
\frac{d y}{d x} \text { in }(4) \leqq \frac{d y}{d x} \text { in (5) } & \text { for } y>0 . \\
\frac{d y}{d x} \text { in }(4) \leqq \frac{d y}{d x} \text { in (6) } & \text { for } y<0 .
\end{array}
$$

LEMMA 1. Any solution of (5) has the following properties in the upper half plane.

(d) It has a positive slope to the left of the curve -cy-g(x)+ $E=0$, a negative slope to the right of this curve, and reaches a maximum of $y$ when crossing the curve.

(e) Any solution starting with $x<x_{2}$ must cross the line $x=x_{2}$ for positive value.

(f) To the right of the line $x=x_{2}$ the solution is concave downward. It leaves the upper half plane as $t$ increases with $x>x_{2}$.

A similar statement holds for the equation (6).

Our proof is similar to that of Loud [4] and is omitted.

Let $y_{1}$ be a positive number. Let $C_{1}$ be the solution of the equation (5) starting from $\left(x_{2}, y_{1}\right)$ to its next intersection with the $x$-axis at $\left(x_{3}, 0\right)$, and let $C_{2}$ be the solution of the equation (6) starting from $\left(x_{3}, 0\right)$ to its next intersection with the line $x=x_{1}$ at $\left(x_{1},-y\right)$. The existence of all the intersections mentioned above is guaranteed by Lemma 1 .

LEMMA 2. Let $C$ be the arc consisting of $C_{1}$ and $C_{2}$. If $y_{1} \geqq 4 E / c$, then $|y|<y_{1}$ at any point of $C$ other than $\left(x_{2}, y_{1}\right)$.

Our proof is a slight modification of that of Loud [4] and is omitted.

Let $B_{1}$ be the solution of (5) starting from $\left(x_{2}, 4 E / c\right)$ to its next intersection with the $x$-axis at $\left(a_{1}, 0\right)$. Let $B_{2}$ be the solution of (6) starting from $\left(a_{1}, 0\right)$ to its next intersection with the line $x=x_{1}$ at $\left(x_{1}, b_{1}\right)$. Then $b_{1}>-4 E / c$ by Lemma 2 . Let $B_{3}$ be the line segment of $x=x_{1}$ from $\left(x_{1}, b_{1}\right)$ to $\left(x_{1},-4 E / c\right)$.

Let $B_{4}$ be the solution of (6) starting from $\left(x_{1},-4 E / c\right)$ to its next intersection with the $x$-axis at $\left(a_{2}, 0\right)$. Let $B_{5}$ be the solution of (5) starting from $\left(a_{2}, 0\right)$ to its next intersection with the line $x=x_{2}$ at $\left(x_{2}, b_{2}\right)$. 
Then $b_{2}<4 E / c$ by the latter half of Lemma 2 . Let $B_{6}$ be the line segment of $x=x_{2}$, from $\left(x_{2}, b\right)$ to $\left(x_{2}, 4 E / c\right)$.

Then the curve $B$ consisting of these six curves $B_{i}(1 \leqq i \leqq 6)$ surrounds the closed region $D$, which is homeomorphic to a disk. (Cf. Fig. 1)

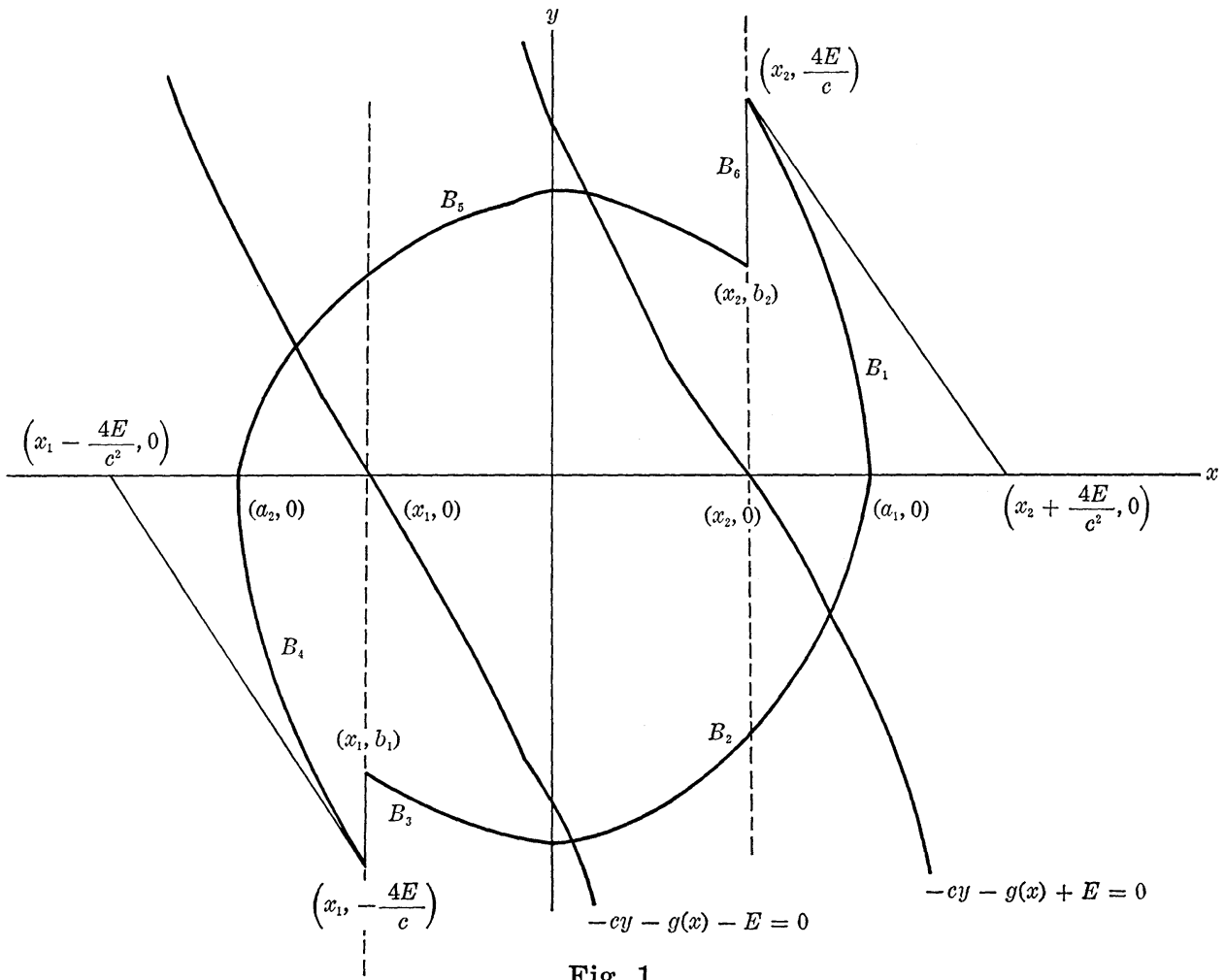

Fig. 1

Using the inequalities (7), (8) and the similar argument in the proof of Theorem 1 of Loud [4], we can prove that any solution of (4) starting outside $D$ enters into $D$ and never leaves $D$ afterward.

By Lemma $2|y| \leqq 4 E / c$ for any point of $D$. And at $\left(x_{2}, 4 E / c\right)$, $\frac{d y}{d x}=-c$ by (5), and the boundary of $D$ is concave downward by Lemma $1(f)$. Therefore, it is easy to see that $D$ is contained in the rectangle $\left[x_{1}-4 E / c^{2}, x_{2}+4 E / c^{2}\right] \times[-4 E / c, 4 E / c]$. This completes the proof of Theorem 1 .

COROLlaRY. In addition to the conditions (a), (b) and (c) of Theorem 1 , we assume the following two conditions $(\mathrm{g})$ and $(\mathrm{h})$. 
(g) $f(x)$ and e(t) are continuous, and $f(x)$ satisfies the local Lipschitz condition.

(h) $e(t)$ is periodic of period $\tau(\tau>0)$.

Then the equation (1) has a periodic solution of period $\tau$.

Proof. By the assumption (g), there exists a unique solution $x=$ $\psi_{1}\left(t ; x_{0}, y_{0}\right), y=\psi_{2}\left(t ; x_{0}, y_{0}\right)$ of (3) satisfying the condition

$$
\left(\psi_{1}\left(0 ; x_{0}, y_{0}\right), \psi_{2}\left(0 ; x_{0}, y_{0}\right)\right)=\left(x_{0}, y_{0}\right)
$$

for each $\left(x_{0}, y_{0}\right) \in \boldsymbol{R}^{2}$. Let $T: \boldsymbol{R}^{2} \rightarrow \boldsymbol{R}^{2}$ be a map defined by

$$
T\left(x_{0}, y_{0}\right)=\left(\psi_{1}\left(\tau ; x_{0}, y_{0}\right), \psi_{2}\left(\tau ; x_{0}, y_{0}\right)\right) .
$$

We call this map $T$ the Poincaré transformation associated with the periodic system (1) (or (3)).

By the assumption (h) and the uniqueness of the solution of (3), it is easy to verify the following equality for each $t$.

$$
\left(\psi_{1}\left(t+\tau ; x_{0}, y_{0}\right), \psi_{2}\left(t+\tau ; x_{0}, y_{0}\right)\right)=\left(\psi_{1}\left(t ; T\left(x_{0}, y_{0}\right)\right), \psi_{2}\left(t ; T\left(x_{0}, y_{0}\right)\right)\right.
$$

Therefore, each fixed point of $T$ defines a periodic solution of period $\tau$ of (1) and vice versa.

By the proof of Theorem $1 T$ maps the closed region $D$ into $D$, and $D$ is homeomorphic to a disk. Therefore, there exists a fixed point of $T$ by the Brouwer's fixed point theorem. This completes the proof of this corollary.

\section{§. The convergence theorem}

In this section we shall prove a convergence theorem for the equations of type (2) with periodic $e(t)$. The equation (2) is equivalent to the following equation.

$$
\left\{\begin{array}{l}
x^{\prime}=y \\
y^{\prime}=-c y-g(x)+e(t)
\end{array}\right.
$$

Throughout this section we assume the following conditions.

A (i) $e(t)$ is a continuous periodic function of period $\tau(\tau>0)$, and $E$ is a positive constant such that $|e(t)| \leqq E$. The existence of such $E$ is guaranteed by our assumption.

A (ii) $g(x)$ is a differentiable function of class $C^{1}$ such that $g^{\prime}(x) \geqq 0$, 
$\lim _{x \rightarrow \infty} g(x)>E, \lim _{x \rightarrow-\infty} g(x)<-E$, and $g^{\prime}(x)=0$ only on a countable subset of the real numbers.

A (iii) $c$ is a positive constant.

By A (ii) the following property is clear.

Property 1. $g(x)$ is strictly monotone increasing, and there exist numbers $x_{1}$ and $x_{2}\left(x_{1}<x_{2}\right)$ such that

$$
g\left(x_{1}\right)=-E \text { and } g\left(x_{2}\right)=E .
$$

Under the assumptions A (i), A (ii) and A (iii) we get the following properties by Theorem 1 and its Corollary.

Property 2. There exists at least one periodic solution of period $\tau$ for the equation (11).

Property 3. Any periodic solution $x=\psi(t), y=\psi_{2}(t)$ of the equation (11) satisfies the following inequalities for all $t$.

$$
\begin{gathered}
x_{1}-4 E / c^{2} \leqq \psi_{1}(t) \leqq x_{2}+4 E / c^{2}, \\
\left|\psi_{2}(t)\right| \leqq 4 E / c .
\end{gathered}
$$

Set $H(\alpha)=\sup \left\{g^{\prime}(x) ;-\alpha \leqq x \leqq \alpha\right\}$ for any positive number $\alpha$. By our assumption $\mathrm{A}(\mathrm{ii}), H(\alpha)$ is finite.

THEOREM 2. Assume the conditions A (i), A (ii) and A (iii). Let $n$ be a positive integer and let $x=\psi_{1}(t), y=\psi_{2}(t)$ be a non-constant periodic solution of period $n \tau$ for the equation (11).

Suppose that $\left|\psi_{1}(t)\right| \leqq \beta$ for all $t$ and $c^{2}>H(\beta)$. Then the periodic solution $x=\psi_{1}(t), y=\psi_{2}(t)$ is asymptotically stable.

This is a generalization of Theorem 2 of Loud [4].

Proof. The variation equation corresponding to the periodic solution $x=\psi_{1}(t), y=\psi_{2}(t)$ is given by

$$
\left\{\begin{array}{l}
x^{\prime}=y \\
y^{\prime}=-c y-g^{\prime}\left(\psi_{1}(t)\right) x .
\end{array}\right.
$$

By our assumption, the variation equation (12) is a linear differetial equation with continuous periodic coefficients. Therefore, to prove that $x=\psi_{1}(t), y=\psi_{2}(t)$ is asymptotically stable, it is sufficient to show that all characteristic exponents of the equation (12) have negative real parts 
(Cf. Chap. 13, Th. 1.4 Coddington-Levinson [1]).

Now, the following Lemma 3 is an easy consequence of the Floquet theory and the theory of linear differential equations with constant coefficients.

LEMMA 3. Let $x^{\prime}=P(t) x$ be a linear differential equation, where $x$ is an $n$-dimensional vector and $P(t)$ is a continuous periodic $n \times n$ matrix function with period $\sigma(\sigma>0)$. Then the following three conditions are equivalent.

(a) The identically zero solution is asymptotically stable.

(b) All characteristic exponents have negative real parts.

(c) Any solution approaches to the origin as $t$ tends to infinity.

By Lemma 3, it is sufficient to prove the following Lemma 4 for the completion of Theorem 2 .

LEMMA 4. Under the assumption of Theorem 2, any solution $(x(t)$, $y(t))$ of (12) tends to the origin as $t$ tends to infinity.

Proof of Lemma 4. From the assymption $\left|\psi_{1}(t)\right| \leqq \beta$ and the definition of $H(\beta)$, we have

$$
0 \leqq g^{\prime}\left(\psi_{1}(t)\right) \leqq H(\beta)
$$

Since $\psi_{1}(t)$ is assumed to be non-constant and $g^{\prime}(x)=0$ on a countable subset of $\boldsymbol{R}$, there exists some $t_{1}$ such that $g^{\prime}\left(\psi_{1}\left(t_{1}\right)\right) \neq 0$. Then we have the following Property 4 by the periodicity and countinuity of $g^{\prime}\left(\psi_{1}(t)\right)$.

PROPERTY 4. There exist positive constants $\delta$ and $\gamma$ satisfying the following condition (B).

(B) For any number $t_{2}$ there exists a number $t_{3}\left(t_{3}>t_{2}\right)$ such that

$$
g^{\prime}\left(\psi_{1}(t)\right) \geqq \delta \quad \text { for } t_{3} \leqq t \leqq t_{3}+\gamma
$$

Let $y_{1}$ be any positive number. Now, we shall construct a bounded closed region $D\left(y_{1}\right)$ as follows.

Let $L_{1}$ be the line segment of $y=-c x+y_{1}$ from $P_{1}\left(0, y_{1}\right)$ to $P_{2}\left(y_{1} / c, 0\right)$. Let $L_{2}$ be the line segment of $x=y_{1} / c$ from $P_{2}\left(y_{1} / c, 0\right)$ to $P_{3}\left(y_{1} / c,-H(\beta) y_{1} / c^{2}\right)$. Let $L_{3}$ be the line segment of $y=-H(\beta) y_{1} / c^{2}$ from $P_{3}\left(y_{1} / c,-H(\beta) y_{1} / c^{2}\right)$ to $P_{4}\left(0,-H(\beta) y_{1} / c^{2}\right)$.

Let $L_{4}$ be the line segment of $y=-c x-H(\beta) y_{1} / c^{2}$ from 
$P_{4}\left(0,-H(\beta) y_{1} / c^{2}\right)$ to $P_{5}\left(-H(\beta) y_{1} / c^{3}, 0\right)$. Let $L_{5}$ be the line segment of $x=-H(\beta) y_{1} / c^{3}$ from $P_{5}\left(-H(\beta) y_{1} / c^{3}, 0\right)$ to $P_{6}\left(-H(\beta) y_{1} / c^{3}, H(\beta)^{2} y_{1} / c^{4}\right)$. Let $L_{6}$ be the line segment of $y=H(\beta)^{2} y_{1} / c^{4}$ from $P_{6}=\left(-H(\beta) y_{1} / c^{3}, H(\beta)^{2} y_{1} / c^{4}\right)$ to $P_{7}\left(0, H(\beta)^{2} y_{1} / c^{4}\right)$.

Since $c^{2}>H(\beta)$ by our assumption, $P_{7}$ is below $P_{1}$ on the $y$-axis. Let $L_{7}$ be the line segment of $x=0$ from $P_{7}\left(0, H(\beta)^{2} y_{1} / c^{4}\right)$ to $P_{1}\left(0, y_{1}\right)$.

Then the polygonal curve $L$ consisting of $L_{i}(1 \leqq i \leqq 7)$ is a closed curve, and the closed region $D\left(y_{1}\right)$ is defined to be the region bounded by $L$. (Fig. 2)

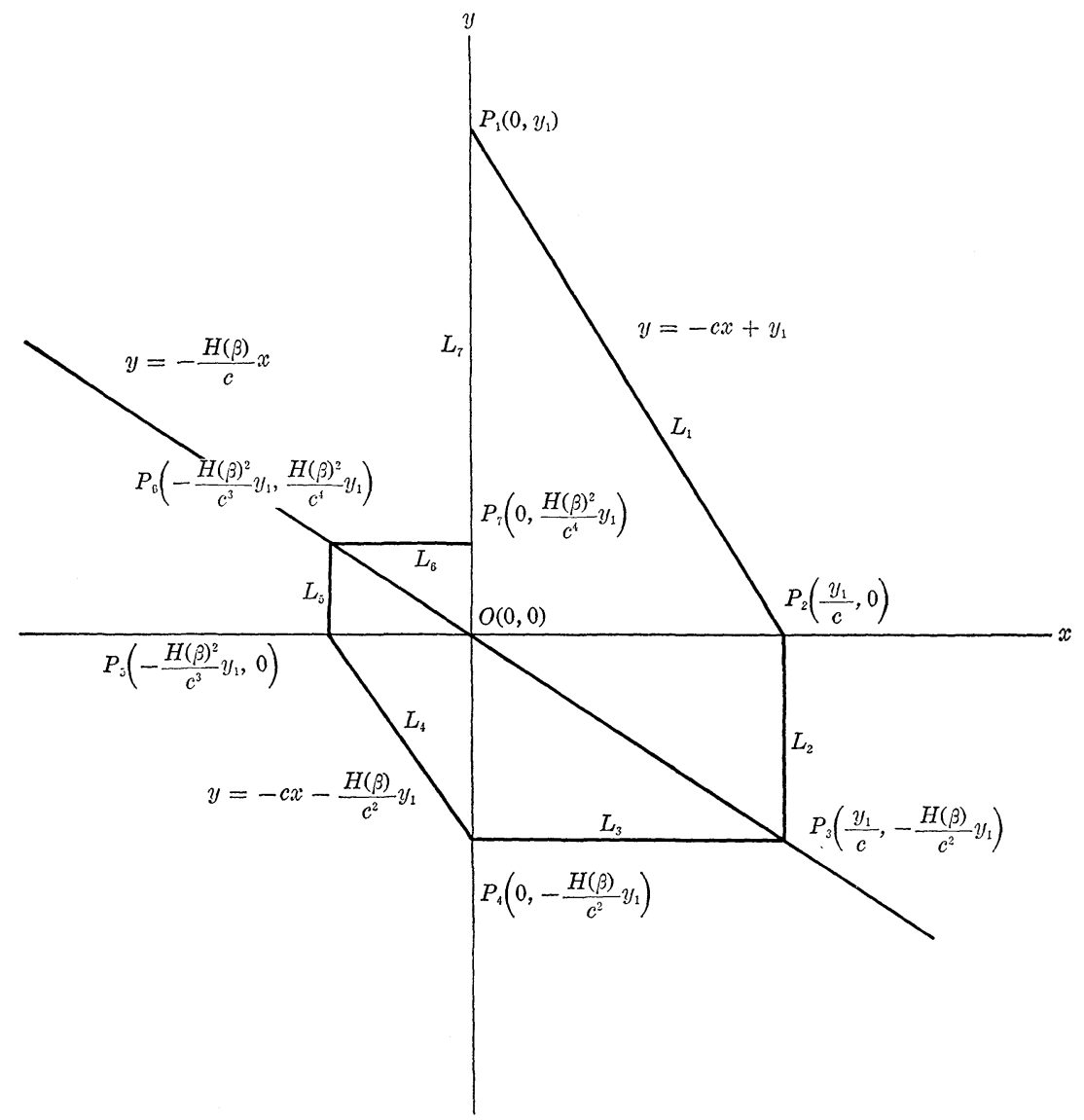

Fig. 2

Using the inequality (13), it is easy to verify that the vector field defined by the equation (12) always points either inside of $D\left(y_{1}\right)$ or tangent to its boundary $L$ on any point of $L$. Therefore, any solution (12) 
starting from the point of $D\left(y_{1}\right)$ at any time $t_{0}$ stays on $D\left(y_{1}\right)$ for any $t \geqq t_{0}$. Thus, the origin $O(0,0)$ is a stable singularity of the equation (12).

Now, we shall prove the following properties.

Property 5. Any solution of (12) starting from a point of the triangle $O P_{1} P_{2}$ except the line segment $O P_{2}$ reaches to the positive half of the $x$-axis in a finite time interval.

PROPERTy 6. Any solution of (12) starting from a point of the rectangle $\mathrm{OP}_{2} \mathrm{P}_{3} \mathrm{P}_{4}$ either reaches to the negative half of the $y$-axis in a finite time interval or stays on the rectangle $O P_{2} P_{3} P_{4}$ and tends to the origin as tends to infinity.

From Properties 5, 6 and the similar properties for the solutions of (12) starting from the points of the triangle $O P_{4} P_{5}$ and the rectangle $O P_{5} P_{6} P_{7}$, we can easily prove Lemma 3 , and this finishes the proof of Theorem 2.

Proof of Property 5. It is easy to see that any solution $x=x(t)$, $y=y(t)$ of (12) starting from a point of the triangle $O P_{1} P_{2}$ except the line segment $O P_{2}$ can leave the triangle $O P_{1} P_{2}$ only from the line segment $O P_{2}$. Since $x^{\prime}(t)=y(t)>0$ as far as $(x(t), y(t)$ ) stays on the triangle $O P_{1} P_{2}$ except the line segment $O P_{2}$, we can assume, without loss of generality, that there exists a positive number $x_{0}$ such that $x(t) \geqq x_{0}>0$ as far as $(x(t), y(t))$ stays on the triangle $O P_{1} P_{2}$.

Suppose that the solution $(x(t), y(t))$ stays on the triangle $O P_{1} P_{2}$ for any $t \geqq t_{1}$. Then using the condition (B) and (12), we have $y^{\prime}(t) \leqq$ $-\delta x_{0}<0$ for infinitely many time intervals of length at least $\gamma$. But this contradicts the boundedness of $y(t)$ which follows immediately from our assumption that $(x(t), y(t))$ stays on the triangle $O P_{1} P_{2}$ for $t \geqq t_{1}$. This completes the proof of Property 5 .

Proof of Property 6. It is easy to verify that any solution $(x(t), y(t))$ of (12) starting from a point of the rectangle $O P_{2} P_{3} P_{4}$ can leave the rectangle $O P_{2} P_{3} P_{4}$ only from the side $O P_{4}$. Therefore, any solution of (12) starting from a point of the rectangle $O P_{2} P_{3} P_{4}$ leaves the fourth quadrant if and only if it leaves the rectangle $O P_{2} P_{3} P_{4}$.

Suppose that $\left(x\left(t_{0}\right), y\left(t_{0}\right)\right)$ belongs to the rectangle $O P_{2} P_{3} P_{4}$ but not 
the origin. Let $K$ be the supremum of the numbers $t_{1}$ such that for $t_{0} \leqq t \leqq t_{1}$ the solution $(x(t), y(t))$ stays on the rectangle $O P_{2} P_{3} P_{4}$.

Case 1. Let $K$ be finite. Then it is clear that $(x(K), y(K))$ belongs to the rectangle $O P_{2} P_{3} O_{4}$. Now, we shall prove that $x(K)=0$ and $y(K)<0$. Thus, the solution $(x(t), y(t))$ reaches the negative half of the $y$-axis in a finite time interval and gets out of the rectangle $O P_{2} P_{3} P_{4}$ there.

By the definition of $K$ and the equation (12), it is easy to see that $x(t)$ is monotone decreasing for $t_{0} \leqq t \leqq K, x(K) \geqq 0$, and $y(K) \leqq 0$.

Suppose that $x(K)>0$. If $y(K)<0$, then $x^{\prime}(K)=y(K)<0$. And it is easy to see that the solution $(x(t), y(t))$ stays on the fourth quadrant for $K \leqq t \leqq K+k_{0}$ for a suitable positive $k_{0}$. This contradicts the definition of $K$. Therefore, $y(K)=0$.

If $y(K)=0$, then $x^{\prime}(K)=y(K)=0$ and $y^{\prime}(K)=-g^{\prime}\left(\psi_{1}(t)\right) x(K) \leqq 0$ by our assumption. Using (13) and Property 4, we easily see that the solution $(x(t), y(t))$ stays on the fourth quadrant for $K \leqq t \leqq K+k_{1}$ for a suitable positive $k_{1}$. But this contradicts the definition of $K$ and establishes that $x(K)=0$.

Next, if $y(K)=0$, then $(x(K), y(K))=(0,0)$. Now, by the uniqueness of the solution of (12) under the same initial condition, $(x(t), y(t))=(0,0)$ for all $t$. This contradicts our assumption that $\left(x\left(t_{0}\right), y\left(t_{0}\right)\right) \neq(0,0)$. Therefore, $y(K)<0$.

Case 2. Let $K=\infty$. Then the solution $(x(t), y(t))$ stays on the rectangle $O P_{2} P_{3} P_{4}$ for all $t \geqq t_{0}$, and $x(t)$ is monotone decreasing for $t \geqq t_{0}$ since $x^{\prime}(t)=y(t) \leqq 0$.

In this case, we shall show that $\lim _{t \rightarrow \infty} x(t)=\lim _{t \rightarrow \infty} y(t)=0$,

Since $x(t)$ is monotone decreasing and $x(t) \geqq 0$ for $t \geqq t_{0}, x(t)$ converges to $x_{0} \geqq 0$ as $t$ tends to infinity.

Suppose that $x_{0}>0$. Since $(x(t), y(t))$ stays on the rectangle $O P_{2} P_{3} P_{4}$ for $t \geqq t_{0}$, there exists a sequence $\left\{t_{k}\right\}$ such that

$$
\lim _{k \rightarrow \infty} t_{k}=\infty \quad \text { and } \lim _{k \rightarrow \infty} y\left(t_{k}\right)=y_{0} \quad \text { for some } y_{0} \leqq 0
$$

Then $\lim _{k \rightarrow \infty} x\left(t_{k}\right)=x_{0}$ since $\lim _{t \rightarrow \infty} x(t)=x_{0}$. Thus, the following property is proved. 
PROPERTY 7. $\lim _{k \rightarrow \infty} t_{k}=\infty, \lim _{k \rightarrow \infty} x\left(t_{k}\right)=x_{0} \geqq 0, x(t)$ is monotone decreasing for $t \geqq t_{0}, x(t) \geqq x_{0}$ for $t \geqq t_{0}$ and $\lim _{k \rightarrow \infty} y\left(t_{k}\right)=y_{0} \leqq 0$.

And we are now assuming that $x_{0}>0$. Further, we assume that $y_{0}<0$. Then there exists a positive number $\ell$ such that $y_{0} / x_{0}<-\ell$.

Let $\varepsilon$ be a positive number, and we put $Q_{1}=\left(x_{0}+\varepsilon, y_{0}\right), Q_{2}=\left(x_{0}\right.$, $\left.y_{0}+c \varepsilon\right)$ and $Q_{3}=\left(x_{0}, y_{0}+(-c+H(\beta) / \ell) \varepsilon\right)$. Then the slope of $Q_{1} Q_{2}$ is $-c$, and the slope of $Q_{1} Q_{3}$ is $-c+H(\beta) / \ell$. Since the point $\left(x_{0}, y_{0}\right)$ is below the line $y=-\ell x$ by our assumption, the triangle $Q_{1} Q_{2} Q_{3}$ is also below the line $y=-\ell x$ for a small $\varepsilon$. We choose such $\varepsilon$. (Fig. 3)

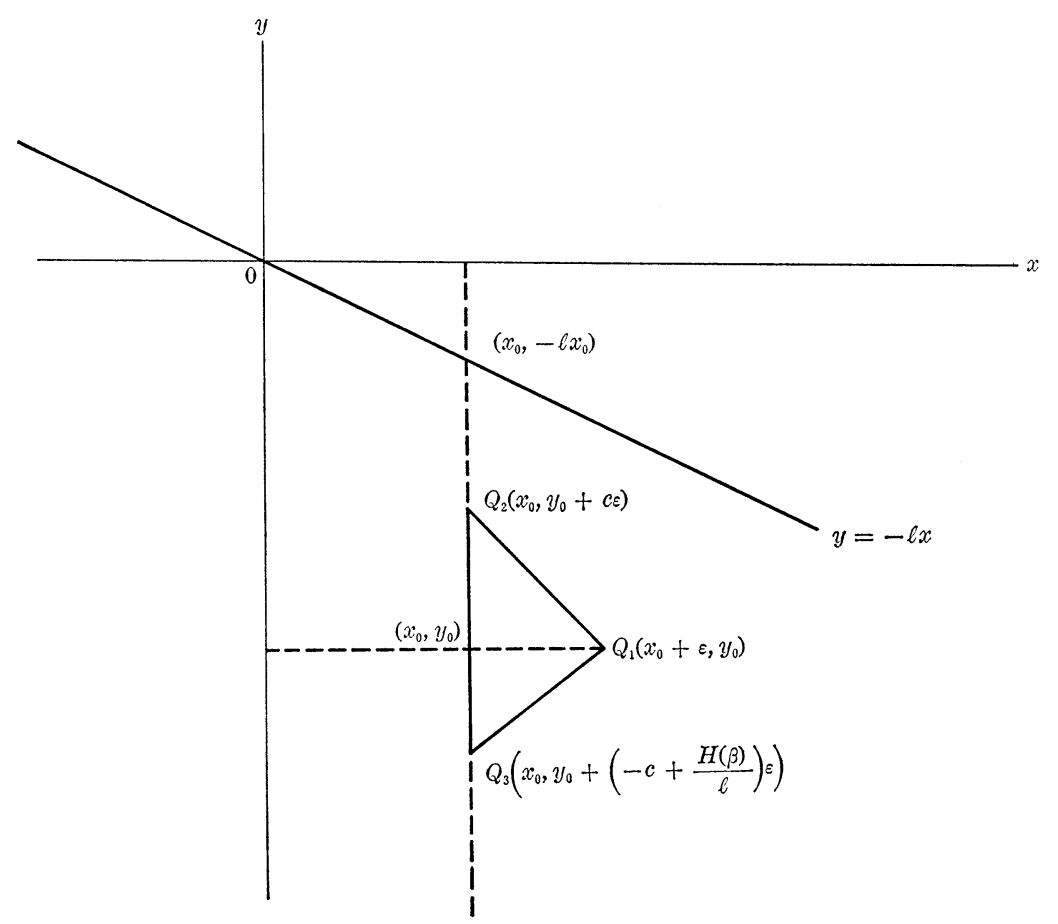

Fig. 3

If $(x, y)$ is below the line $y=-\ell x$ and is in the rectangle $O P_{2} P_{3} P_{4}$, then by using (13) it is easy to see that any solution of (12) satisfies the following inequalities at the point $(x, y)$.

$$
-c \leqq \frac{d y}{d x}=-c-g\left(\psi_{1}(t)\right) \frac{x}{y} \leqq-c+H(\beta) / \ell
$$

By (14) and the definition of the triangle $Q_{1} Q_{2} Q_{3}$, we conclude that 
any solution of (12) starting from a point in the triangle $Q_{1} Q_{2} Q_{3}$ can leave it only from its side $Q_{2} Q_{3}$. By Property 7 , there exists a $t_{k}$ such that $\left(x\left(t_{k}\right), y\left(t_{k}\right)\right)$ lies in the triangle $Q_{1} Q_{2} Q_{3}$. Therefore, $(x(t), y(t))$ must stay in the triangle $Q_{1} Q_{2} Q_{3}$ for all $t \geqq t_{k}$.

By (12) and the construction of the triangle $Q_{1} Q_{2} Q_{3}, x^{\prime}(t)=y(t) \leqq$ $y_{0}+c \varepsilon<-\ell x_{0}<0$ for $t \geqq t_{k}$. Therefore, $x(t)$ cannot be bounded below. This contradicts to $\lim _{t \rightarrow \infty} x(t)=x_{0}$.

By the above argument, $y_{0}=0$.

By the same argument as above, we can prove the following property.

PROPERTy 8. Let $\left\{t_{k}\right\}$ be any sequence such that $\lim _{k \rightarrow \infty} t_{k}=\infty$ and $y\left(t_{k}\right)$ converges as $k$ tends to infinity. Then $\lim _{k \rightarrow \infty} y\left(t_{k}\right)=0$.

Therefore, $\lim _{t \rightarrow \infty} y(t)$ exists and equal to 0 . Thus, we have obtained the following equalities.

$$
\lim _{t \rightarrow \infty} x(t)=x_{0}>0, \quad \lim _{t \rightarrow \infty} y(t)=0
$$

Let $\varepsilon$ be a positive number such that

$$
\varepsilon \leqq \min \left\{\delta x_{0} /(2 c), \delta x_{0} \gamma / 2\right\},
$$

where $\delta$ and $\gamma$ are given in Property 4 .

By (15) there exists a number $\bar{t}$ such that

$$
\left|x(t)-x_{0}\right|<\varepsilon \text { and }|y(t)|<\varepsilon \quad \text { for } t \geqq \bar{t} .
$$

By Property 7 and (17), it follows that

$$
0<x_{0} \leqq x(t)<x_{0}+\varepsilon \quad \text { and } \quad-\varepsilon<y(t) \leqq 0 \quad \text { for } t \geqq \bar{t} .
$$

By condition (B) of Property 4, there exists a number $\bar{t}_{1} \geqq \bar{t}$ such that $g^{\prime}\left(\psi_{1}(t)\right) \geqq \delta$ for $\bar{t}_{1} \leqq t \leqq \bar{t}_{1}+\gamma$. Therefore, by (16) and (18) we have the following inequalities for $\bar{t}_{1} \leqq t \leqq \bar{t}_{1}+\gamma$.

$$
\begin{aligned}
y^{\prime}(t)= & -c y(t)-g^{\prime}\left(\psi_{1}(t)\right) x(t) \\
& <c \varepsilon-\delta x_{0} \leqq c \delta x_{0} /(2 c)-\delta x_{0} \\
& =-\delta x_{0} / 2
\end{aligned}
$$

By the mean value theorem, (16), (18) and (19), we have the following inequalities. 


$$
y\left(\bar{t}_{1}+\gamma\right)<y\left(\bar{t}_{1}\right)-\left(\delta x_{0} / 2\right) \gamma \leqq-\delta x_{0} \gamma / 2 \leqq-\varepsilon
$$

But this contradicts to the latter half of (18).

By the above, we conclude that $x_{0}=0$, that is $\lim _{t \rightarrow \infty} x(t)=0$.

Now, using a similar argument as above, we can prove that $\lim _{t \rightarrow \infty} y(t)$ exists and is equal to 0 .

Thus, we finish the proof of Property 6, and this completes the proof of Theorem 2 .

Corollary 1. Assume the conditions A (i), A (ii) and A (iii). Let $A=\max \left\{\left|x_{1}-4 E / c^{2}\right|,\left|x_{2}+4 E / c^{2}\right|\right\}$, where $x_{1}$ and $x_{2}$ satisfy the equation $g\left(x_{1}\right)=-E$ and $g\left(x_{2}\right)=E$. Further, assume that $c^{2}>H(A)$.

Then every non-constant periodic solution of period $n \tau$ ( $n$ a positive integer) of the equation (11) is asymptotically stable.

Proof. This is an immediate consequence of Theorem 2 and Property 3.

COROLlaRY 2. In addition to the assumptions of Corollary 1, we assume that $e(t)$ is non-constant. Then there exists a non-constant periodic solution $x=\psi_{1}(t), y=\psi_{2}(t)$ of period $\tau$ for the equation (11) such that any periodic solution of period $n \tau$ (for a suitable positive integer $n$ ) for the equation (11) coincides with the solution $x=\psi_{1}(t), y=\psi_{2}(t)$.

Proof. By Property 2 there exists a periodic solution $x=\psi_{1}(t)$, $y=\psi_{2}(t)$ of period $\tau$ for the equation (11). Since $e(t)$ is non-constant by our assumption, there is no constant solution for the equation (11). Therefore, the periodic solution $x=\psi_{1}(t), y=\psi_{2}(t)$ is non-constant, and it may be considered as a periodic solution of period $n \tau$ for the equation (11) for an arbitrary positive integer $n$.

Let $T: \boldsymbol{R}^{2} \rightarrow \boldsymbol{R}^{2}$ be the Poincaré transformation associated with the equation (11). Then there is a one-to-one correspondence between the set of the periodic solution of period $n \tau$ for the equation (11) and the set of the fixed points of $T^{n}$ ( $n$ times iterate of $T$ ).

By Corollary 1, every periodic solution of period $n \tau$ is asymptotically stable. Therefore, every fixed point of $T^{n}$ is completely stable, and it is contained in a compact region $D$ defined in the proof of Theorem 1 . Since a completely stable fixed point is isolated, there are only a finite number of fixed points of $T^{n}$ for a fixed $n$. 
The index of a completely stable fixed point is +1 , and the sum of all the indices of the fixed points of $T^{n}$ is equal to 1 for every $n$. Therefore, there exists only one fixed point of $T^{n}$ for each fixed $n$. This must correspond to the periodic solution $x=\psi_{1}(t), y=\psi_{2}(t)$. This completes the proof of Corollary 2.

THEOREM 3. Under the same assumption of Corollary 2 of Theorem 2 , there exists a unique periodic solution $x=\psi_{1}(t), y=\psi_{2}(t)$ of period $\tau$ for the equation (11) such that for any solution $x=x(t), y=y(t)$ of (11) the following equalities hold.

$$
\lim _{t \rightarrow \infty}\left|x(t)-\psi_{1}(t)\right|=\lim _{t \rightarrow \infty}\left|y(t)-\psi_{2}(t)\right|=0
$$

That is any solution of (11) converges to a unique periodic solution of period $\tau$ of (11).

Proof. Let $x=\psi_{1}(t), y=\psi_{2}(t)$ be the unique periodic solution of period $\tau$ for the equation (11), and let $x=x(t), y=y(t)$ be any solution of (11). Set $z(t)=x(t)-\psi_{1}(t)$.

Since $x(t)$ and $\psi_{1}(t)$ are solutions of the equation (2), $z(t)$ satisfies the following equation.

$$
z^{\prime \prime}+c z^{\prime}+g^{\prime}(\delta(t)) z=0
$$

where $g^{\prime}(\delta(t)) z(t)=g(x(t))-g\left(\psi_{1}(t)\right)$ and $\delta(t)$ is a number between $x(t)$ and $\psi_{1}(t)$ for each $t$.

By our assumption $g^{\prime}(\delta(t))$ satisfies the following inequality.

$$
0 \leqq g^{\prime}(\delta(t)) \leqq H(A) \quad \text { for sufficiently large } t
$$

When Property 4 holds for $g^{\prime}(\delta(t))$, then we can prove that $\lim _{t \rightarrow \infty} z(t)$ $=\lim _{t \rightarrow \infty} z^{\prime}(t)=0$ as in the proof of Lemma 4. Therefore, it is sufficient to prove our theorem in case that Property 4 does not hold.

In this case, for any positive numbers $\delta$ and $\gamma$, there exists a number $t_{1}$ such that for any $t_{2}>t_{1}$ there exists a number $t_{3}\left(t_{2} \leqq t_{3} \leqq t_{2}+\gamma\right)$ such that $g^{\prime}\left(\delta\left(t_{3}\right)\right)<\delta$. Therefore, there exists a sequence $\left\{t_{k}\right\}$ such that

$$
\mathrm{ilm}_{k \rightarrow \infty} t_{k}=\infty \text { and } \lim _{k \rightarrow \infty} g^{\prime}\left(\delta\left(t_{k}\right)\right)=0 .
$$

By the equality $g^{\prime}(\delta(t)) z(t)=g(x(t))-g\left(\psi_{1}(t)\right)$ and the boundedness 
of $z(t)=x(t)-\psi_{1}(t)$ for sufficiently large $t$, we have $\lim _{k \rightarrow \infty}\left(g\left(x\left(t_{k}\right)\right)-\right.$ $\left.g\left(\psi_{1}\left(t_{k}\right)\right)\right)=0$.

By our assumption, $|x(t)| \leqq A,\left|\psi_{1}(t)\right| \leqq A$ for sufficiently large $t$, and $g$ is a homeomorphism between $[-A, A]$ and $[g(-A), g(A)]$. Therefore, $g^{-1} \mid[g(-A), g(A)]$ is uniformly continuous, and we can conclude that $\lim _{k \rightarrow \infty}\left(x\left(t_{k}\right)-\psi_{1}\left(t_{k}\right)\right)=0$.

The equation (20) is equivalent to the following equation.

$$
\left\{\begin{array}{l}
z^{\prime}=u \\
u^{\prime}=-c u-g^{\prime}(\delta(t)) z
\end{array}\right.
$$

By a similar argument as in the first part of the proof of Lemma 4 using (21), we can prove that any solution of (22) is bounded for large $t$. Therefore, there exists a subsequence $\left\{t_{k}^{\prime}\right\}$ of $\left\{t_{k}\right\}$ such that $\left\{u\left(t_{k}^{\prime}\right)\right\}$ converges as $k$ tends to infinity. Put $\lim _{k \rightarrow \infty} u\left(t_{k}^{\prime}\right)=u_{0}$.

Since $\lim _{k \rightarrow \infty} z\left(t_{k}\right)=\lim _{k \rightarrow \infty}\left(x\left(t_{k}\right)-\psi_{1}\left(t_{k}\right)\right)=0$, we have $\lim _{k \rightarrow \infty} z\left(t_{k}^{\prime}\right)=0$. Now, we shall prove that $u_{0}=0$, that is $\lim _{k \rightarrow \infty} u\left(t_{k}^{\prime}\right)=0$.

Suppose that $u_{0} \neq 0$, say $u_{0}>0$. Then, using a similar construction as in the first part of the proof of Lemma 4, we can prove the following property.

Property 9. There exists a positive number $\mu$ such that for any solution $z=z(t), u=u(t)$ of the equation (22) starting from a point sufficiently near the point $\left(0, u_{0}\right)$ the following inequality holds.

$$
u(t) \leqq u_{0}-\mu \quad \text { for sufficiently large } t .
$$

But this contradicts our assumption $\lim _{k \rightarrow \infty} u\left(t_{k}^{\prime}\right)=u_{0}$.

The case $u_{0}<0$ also leads to a contradiction. Thus, $u_{0}=0$. Therefore, we have proved that there exists a sequence $\left\{t_{k}^{\prime}\right\}$ such that

$$
\lim _{k \rightarrow \infty} z\left(t_{k}^{\prime}\right)=\lim _{k \rightarrow \infty} u\left(t_{k}^{\prime}\right)=0 \text {. }
$$

By Corollary 2 of Theorem 2, $x=\psi_{1}(t), y=\psi_{2}(t)$ is an asymptotically stable periodic solution of (11). Therefore, any solution of (11) sufficiently near to the solution $\left(\psi_{1}(t), \psi_{2}(t)\right)$ for some $t$ must converge to the solution $\left(\psi_{1}(t), \psi_{2}(t)\right)$ as $t$ tends to infinity. And (23) is equivalent to the following (24). 


$$
\lim _{k \rightarrow \infty}\left(x\left(t_{k}^{\prime}\right)-\psi_{1}\left(t_{k}^{\prime}\right)\right)=\lim _{k \rightarrow \infty}\left(y\left(t_{k}^{\prime}\right)-\psi_{2}\left(t_{k}^{\prime}\right)\right)=0
$$

This completes the proof.

\section{REFERENCES}

[1] Coddington-Levinson: Theory of ordinary differential equations, McGraw-Hill (1955).

[2] C. Hayashi, Y. Ueda and H. Kawakami: Transformation theory as applied to the solution of non-linear differential equations of the second order, Int. J. NonLinear Mechanics, 4 (1969), 235-255.

[3] H. Kawakami: Qualitative study on the solutions of Duffing's equation, Thesis (1973), Kyoto University.

[4] W. S. Loud: Boundedness and convergence of solutions of $x^{\prime \prime}+c x^{\prime}+g(x)=e(t)$, Duke Math. J., 24 (1957), 63-72.

Department of Mathematics

College of General Education

Nagoya University 